\title{
Coronary heart disease and risk factors in NIDDM - experience from the United Kingdom Prospective Diabetes Study
}

\author{
R .C. Turner, H . M illns, R . R . H olman \\ Diabetes Research Laboratories, Oxford University, UK
}

The United Kingdom Prospective Diabetes Study (UKPDS) is a randomised, controlled trial of different therapies for patients with non-insulin-dependent diabetes (NIDDM). The study, which recruited 5102 newly diagnosed diabetic patients between 1977 and 1991 will close out at the end of 1997 [1]. Publication of the main results will be in late 1998 .

The patients, aged 25-65 years inclusive, were recruited from general practitioners in 23 clinical centres and were thus likely to be representative of diabetic patients in the UK. They had a median age of 53 years, with median fasting plasma glucose levels of $12 \mathrm{mmol} / \mathrm{l}$ and median body mass index of $28 \mathrm{~kg}$ $\mathrm{m}^{-2}$. At diagnosis, most were well apart from their diabetes but $8 \%$ of the patients already had clinical evidence of heart disease, with $2 \%$ having had a myocardial infarction, $3 \%$ angina, $1 \%$ stroke or transient ischaemic attack and $3 \%$ intermittent claudication. Those who had had recent heart attacks, angina or heart failure were excluded from the prospective study.

By 9 years follow-up, $29 \%$ had developed a clinical complication, with $20 \%$ having had a macrovascular event viz $13 \%$ myocardial infarction (fatal in $50 \%$ ), $3 \%$ stroke (fatal in $30 \%$ ), $6 \%$ angina with an abnormal ECG, $2 \%$ heart failure. In addition $9 \%$ developed clinically apparent microvascular disease (6\% retinal photocoagulation, $0.7 \%$ vitreous haemorrhage, $0.7 \%$ renal failure).

The risk factors for the development of coronary heart disease were studied in 3055 white Caucasian patients who had no indication of coronary heart disease at diagnosis of diabetes. Within 10 years 335 subjects developed fatal or non-fatal myocardial infarction or angina. In a Cox model adjusting for age and gender,

Corresponding author: Professor R. C. Turner, Diabetes Research Laboratories, Radcliffe Infirmary, Woodstock Road, Oxford OX2 6HE, UK the following variables assessed after 3 months' initial diet therapy were found to be risk factors: a raised cholesterol, LDL cholesterol or triglyceride, a low HDL cholesterol, raised haemoglobin $\mathrm{A}_{1 \mathrm{c}}$ or fasting plasma glucose, systolic or diastolic blood pressure and current smoking. Notable variables not associated with coronary heart disease were: obesity, represented as a raised body mass index or waist-hip ratio; a sedentary lifestyle; and raised insulin concentrations, denoting impaired insulin sensitivity. Thus, although these factors are known to contribute to the development of diabetes and are also risk factors for coronary heart disease, once diabetes developed other factors became more important in regard to developing heart disease. The expected 'deadly quartet', enunciated by Kaplan [2] of upper segment obesity, raised triglyceride levels and hypertension, in addition to hyperglycaemia was not dominant. In a multivariate model the main risk factors were raised LDL cholesterol, low HDL cholesterol, raised haemoglobin $A_{1 c}$, raised systolic blood pressure and current smoking, as in the general population.

The potential influence of microvascular disease was examined by determining whether the presence of microalbuminuria or retinopathy at the diagnosis of diabetes was an additional risk factor when added to the model. However, the model showed no significant contribution from either of these although both have previously been reported as risk factors. Both hyperglycaemia and hypertension have been shown to be the major risk factors for the development of these microvascular diseases in the UKPDS population as well as for coronary heart disease. It is possible that in published studies where microalbuminuria and retinopathy were suggested to be risk factors for heart disease, these microvascular diseases were acting as biological markers for previous hyperglycaemia and hypertension that also increased the susceptibility to coronary heart disease. 
Since coronary heart disease is the major clinical complication of non-insulin-dependent diabetes in the UK, therapy might particularly be directed to prevention of smoking and to minimising hyperglycaemia, hypertension and dyslipidaemia. However, no prospective randomised studies have documented whether clinical benefit will be obtained, whether any particular therapeutic goals are important, or whether any specific pharmaceutical agents provide benefit. Only long-term prospective studies will provide this information. To assess whether improved blood glucose control would be advantageous, the UKPDS has randomised 3866 NIDDM patients who had persistent hyperglycaemia after 3 months' diet therapy with fasting plasma glucose values 6$15 \mathrm{mmol} / \mathrm{l}$, to different therapies. There are two main comparison groups: (i) 1138 patients allocated to a conventional policy, initially with diet, keeping asymptomatic with fasting plasma glucose levels less than $15 \mathrm{mmol} / \mathrm{l}$; and (ii) 2728 patients allocated to an intensive therapy policy increasing insulin supply with sulphonylurea or insulin therapy aiming for fasting plasma glucose less than $6 \mathrm{mmol} / \mathrm{l}$. A maintained fasting plasma glucose and haemoglobin $\mathrm{A}_{1 \mathrm{c}}$ difference has been achieved with median values over 10 years of 9.0 and $7.4 \mathrm{mmol} / \mathrm{l}$ and 7.6 and $6.9 \%$, respectively. In addition, use of metformin and acarbose are also being evaluated. To determine whether improved blood pressure control is advantageous, 1148 diabetic patients with co-existing hypertension have been allocated to the 'Hypertension in Diabetes Study' (HDS) to 'less tight control' and 'tight control' achieving median blood pressure values over 5 years of 154/86 and 141/80 $\mathrm{mmHg}$, respectively.

The UKPDS and HDS will report in 1998, after a median of 11 and 9 year follow-up periods respectively. They will determine whether the improved blood glucose and blood pressure control that can be achieved in clinical practice will decrease the incidence of macrovascular and microvascular clinical complications, and will thus help to maintain the health of patients. It is planned that from 1998, 4000 patients will be recruited into the Lipids in Diabetes study to evaluate whether routine fibrate and/or statin therapy will prevent cardiovascular disease.

\section{References}

1. VKPDS Group (1991) VKPDS VIII: Study design, progress and performance. Diabetologia 34: 877-890

2. Kaplan NM (1989) The deadly quartet. Upper-body obesity, glucose intolerance, hypertriglyceridaemia and hypertension. Arch Int Med 169: 1514-1520 